\title{
ON AN INDUCTION THEOREM FOR RELATIVE GROTHENDIECK GROUPS ${ }^{1}$
}

\author{
WILLIAM H. GUSTAFSON
}

Abstract. We present an improvement in the proof of Dress' induction theorem for relative Grothendieck rings.

1. Introduction. Let $G$ be a finite group, $\mathfrak{U}$ a collection of subgroups of $G$ and $R$ a commutative ring with identity element. Let $\mathfrak{M}$ denote the category of finitely generated (left) modules over the group ring $R G$. For $M \in \operatorname{obj}(\mathfrak{M})$, let $[M]$ denote the isomorphism class of $M$. Let $A$ denote the free abelian group generated by all $[M], M \in \operatorname{obj}(\mathfrak{M})$, and let $B$ denote the subgroup of $A$ generated by all $[M]-\left[M^{\prime}\right]-\left[M^{\prime \prime}\right]$ such that there is an exact sequence

$$
0 \rightarrow M^{\prime} \rightarrow M \rightarrow M^{\prime \prime} \rightarrow 0
$$

in $\mathfrak{M}$ which splits upon restriction to $R H$ for each $H \in \mathfrak{U}$. The quotient group $A / B$ is the relative Grothendieck group of $R G$ relative to $\mathfrak{U}$, denoted by $a_{R}(G, \mathfrak{U})$.

Dress [2] has given some induction theorems for $a_{R}(G, \mathfrak{U})$, in the spirit of Artin's induction theorem for rational characters of $G$. Dress' results depend on a crucial proposition which is proved with some difficulty. In this note, we show that the proposition follows readily from [4], provided that the ground ring $R$ is an algebra (not necessarily faithful) over the ring of $p$-adic integers. Thus our proof is applicable to the important modular case.

2. The main proposition. By a $G$-set, we mean a finite set on which $G$ acts from the left by permutations. Every $G$-set can be written uniquely as a disjoint union of sub- $G$-sets on which $G$ acts transitively. Further, if $S$ is a transitive $G$-set, then $S$ is isomorphic (in the category of $G$-sets and $G$-equivariant set maps) to a $G$-set of the form $G / / H$, i.e., the collection of left cosets of a subgroup $H$ of $G$, with the natural action of $G$.

For any $G$-set $S$, we may define a finitely generated left $R G$-module $R S$ as follows: $R S$ is the free $R$-module with $S$ as basis, and with action of $G$ determined by the $G$-set structure of $S$. In particular, if $S=G$ with

Received by the editors November 29, 1971.

AMS 1970 subject classifications. Primary 13D15, 18F25, 20C10; Secondary 20C20.

Key words and phrases. Induction theorem, relative Grothendieck group.

${ }^{1}$ Research partially supported by an Indiana University Summer Faculty Fellowship. 
action given by left multiplication, then $R S \cong R G$ as left $R G$-modules. If $S$ consists of one element, with each element of $G$ acting as the identity, then $R S$ is just the trivial representation of $G$ over $R$. The proposition of Dress to which we have alluded above is

Proposition 1 (Dress [2, p. 93]). Let $R$ be any commutative ring with unit, let $G$ be an elementary abelian p-group of type $(p, p)$ and let $H$ be $a$ subgroup of $G$ of order $p$. Then the following relation holds in $a_{R}(G, H)$ $\left(=a_{R}(G,\{H\})\right)$ :

$$
p \cdot[R]+[R G]=\sum_{V}[R(G / / V)],
$$

where the sum on the right extends over all the subgroups $V$ of order $p$ in $G$.

Dress' proof requires a detailed and delicate analysis of the structure of $R G$ - and $R H$-modules.

3. Proposition 1 when $R$ is a $p$-adic algebra. In this section we prove Proposition 1 in the case where $R$ is an algebra over the valuation ring $Z_{p}^{*}$ in the $p$-adic completion of the rationals. It is clear that we may assume that $R=Z_{p}^{*}$, and that $a_{R}(G, H)$ is calculated from the category of $R G$ lattices (i.e. finitely generated left $R G$-modules which are free as $R$ modules). This calculation has been given in detail in [4]; let us recall what was found there. We present $G$ as $\left\langle a, b \mid a^{p}=b^{p}=[a, b]=1\right\rangle$. Without loss of generality, we may take $H=\langle a\rangle$. Denote by $C$ the companion matrix of the cyclotomic polynomial $\Phi_{p}(X)$. Thus

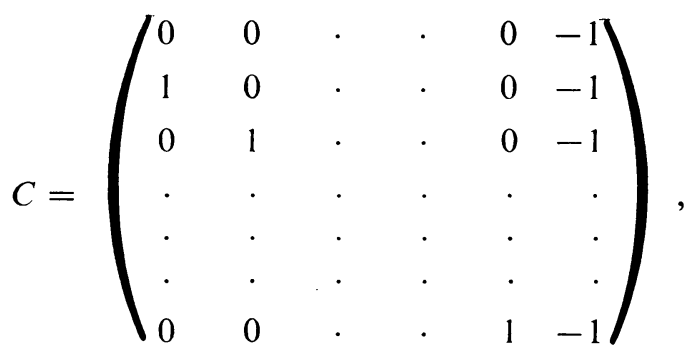

a $(p-1) \times(p-1)$ matrix. The $R$-irreducible $R G$-lattices are the trivial lattice $T \cong R$ and lattices $W, X_{0}, \cdots, X_{n-1}$ which afford matrix representations as follows:

$$
\begin{aligned}
W: a \rightarrow I_{(p-1) \times(p-1)}, & b & \rightarrow C, \\
X_{k}: a \rightarrow C, & b & \rightarrow C^{k}(k=0, \cdots, p-1) .
\end{aligned}
$$

Then $a_{R}(G, H)$ is a free abelian group with basis $[T],[W],\left[X_{0}\right], \cdots$, $\left[X_{p-1}\right],\left[\left(X_{0}, T\right)\right]$, where $\left(X_{0}, T\right)$ is a certain extension of $X_{0}$ by $T$ described 
in detail in [4]. We define certain group homomorphisms as follows:

$$
\begin{aligned}
\pi: a_{R}(G, H) & \rightarrow a_{R}(G,\{1\}), \pi[M]=[M], \\
\lambda: a_{R}(G,\{1\}) & \rightarrow a_{R}(G, H), \lambda[I]=[I] \text { for } I R \text {-irreducible, } \\
\text { res: } a_{R}(G, H) & \rightarrow a_{R}(H, H), \operatorname{res}[M]=\left[M_{H}\right], \\
\varphi: a_{R}(G, H) & \rightarrow a_{R}(G, 1) \oplus a_{R}(H, H), \varphi[M]=(\pi[M], \operatorname{res}[M]) .
\end{aligned}
$$

For any $R G$-lattice $M$, define $t_{M I} \in a_{R}(G, H)$ by

$$
t_{M}=\lambda \pi([M])+v \cdot\left(\left[X_{0}, T\right]-\left[X_{0}\right]-[T]\right),
$$

where $\nu$ is determined by

$$
\operatorname{res}[M]=\nu \cdot[R H]+a \cdot[A]+b \cdot[B],
$$

$A=R$ and $B=R\left[1^{1 / p}\right]$ being the irreducible $R H$-lattices.

Proposition 2. For any $R G$-lattice $M, t_{M}=[M]$ in $a_{R}(G, H)$.

Proof. Assume res $[M]$ is given by equation (1) above. By the proof of Theorem 4.7 of [4], $\varphi$ is a monomorphism. Hence it suffices to show that $\pi[M]=\pi\left(t_{M}\right)$ and $\operatorname{res}[M]=\operatorname{res} t_{M}$.

Suppose that $\pi[M]=t[T]+\sum x_{i}\left[X_{i}\right]+w \cdot[W]$. Then $\lambda \pi[M]$ is given by the same formula, but lies in $a_{R}(G, H)$. Recalling that $T_{I I} \cong A,\left.X_{i}\right|_{H} \cong B$ and $W_{H} \cong A \oplus \cdots \oplus A(p-1$ copies $)$, we see that

$$
M_{H} \cong v \cdot R H+\left(-v+\sum x_{i}\right) B+(t-v+(p-1) w) A .
$$

By (1) and the Krull-Schmidt theorem for $R H$-lattices (see [1, Theorem 76.26]), we have

$$
b=-v+\sum x_{i}, \quad a=t-v+(p-1) w .
$$

We also have

$$
\pi\left(t_{1 Y}\right)=\pi \lambda \pi([M])+v \cdot \pi\left(\left[\left(X_{0}, T\right)\right]-\left[X_{0}\right]-[T]\right)=\pi([M]),
$$

since clearly $\pi\left(\left[\left(X_{0}, T\right)\right]-\left[X_{0}\right]-[T]\right)=0$.

On the other hand,

$$
\text { res } \begin{aligned}
t_{. M} & =\operatorname{res} \lambda \pi[M]+v \cdot \operatorname{res}\left(\left[\left(X_{0}, T\right)\right]-\left[X_{0}\right]-[T]\right) \\
& =\operatorname{res}\left(t[T]+\sum x_{i}\left[X_{i}\right]+w[W]\right)+v \cdot([R H]-[B]-[A]) \\
& =(t-v+(p-1) w)[A]+\left(-v+\sum x_{i}\right)[B]+v \cdot[R H] \\
& =a[A]+b[B]+v[R H]=\operatorname{res}[M]
\end{aligned}
$$

whence the proposition is established. 
COROllary. In $a_{R}(G, H)$, we have

$$
[R G]+p[R]=[T]+(1-p)\left[X_{0}\right]+\sum_{i \neq 0}\left[X_{i}\right]+[W]+p \cdot\left[\left(X_{0}, T\right)\right]
$$

Now we will use Proposition 2 to calculate the right-hand side of the equation in Proposition 1. Let $H_{k}=\left\langle a^{k} b\right\rangle$, for $k=1, \cdots, p$. Then a full set of subgroups of order $p$ is $H, H_{1}, \cdots, H_{p}$. The cosets of $H$ are $H, b H$, $b^{2} H, \cdots, b^{p-1} H$, from which we see easily that $R(G / / H)$ affords the matrix representation

$$
a \rightarrow I_{p \times p}, \quad b \rightarrow\left(\begin{array}{ccccccc}
0 & 0 & . & . & . & 0 & 1 \\
1 & 0 & . & . & . & 0 & 0 \\
0 & 1 & . & . & . & 0 & 0 \\
. & . & . & . & . & . & . \\
. & . & . & . & . & . & . \\
. & . & . & . & . & . & . \\
0 & 0 & . & . & . & 1 & 0
\end{array}\right) .
$$

By a change of basis, this representation may be brought to the form

$$
a \rightarrow I_{p \times p}, \quad b \rightarrow\left(\begin{array}{c|c}
1 & * \\
\hline 0 &
\end{array}\right) .
$$

Hence we have

$$
\operatorname{res}[R(G / H)]=p[A], \quad \lambda \pi[R(G / / H)]=[T]+[W] .
$$

The cosets of $H_{k}$ are $H_{k}, a H_{k}, \cdots, a^{p-1} H_{k}$, and one has $a^{i} b^{j} \in a^{i-k j} H_{k}$. From this it follows that $R\left(G / / H_{k}\right)$ affords the matrix representation $a \rightarrow \Gamma, b \rightarrow \Gamma^{p-k}$ where

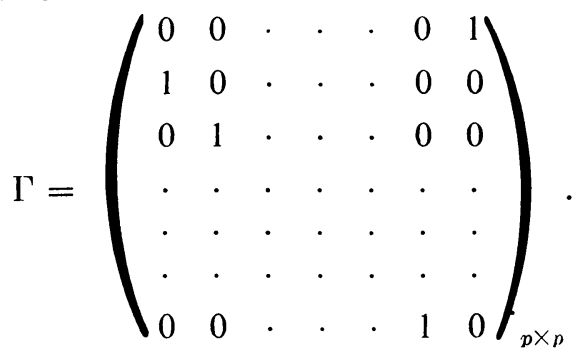

By change of basis, $\Gamma$ may be transformed to

$$
\left(\begin{array}{c|c}
1 & * \\
\hline 0 & -
\end{array}\right)
$$


and the same basis change transforms $\Gamma^{p-k}$ to

Thus we see that

$$
\left(\begin{array}{c|c}
1 & { }^{* \prime} \\
\hline 0 & C^{p-k}
\end{array}\right) \text {. }
$$

$$
\operatorname{res}\left[R\left(G / / H_{k}\right)\right]=[R H], \quad \lambda \pi\left[R\left(G / / H_{k}\right)\right]=[T]+\left[X_{p-k}\right] .
$$

Thus it follows from Proposition 2 that

$$
\begin{aligned}
\sum_{|V|=p}[R(G)]= & {[T]+[W]+p \cdot\left(\left[\left(X_{0}, T\right)\right]-\left[X_{0}\right]-[T]\right) } \\
& +p[T]+\sum_{i=0}^{n-1}\left[X_{i}\right] \\
= & {[T]+[W]+p\left[\left(X_{0}, T\right)\right]+(1-p)\left[X_{0}\right] } \\
& +\sum_{i \neq 0}\left[X_{i}\right] .
\end{aligned}
$$

We may now deduce Proposition 1 by comparing equation (2) and the Corollary to Proposition 2.

4. Descending to the integers. It would be pleasing to use this method to obtain the full force of Proposition 1. In order to do so, it would suffice to handle the case in which $R$ is the ring $Z$ of rational integers. Dress [3, Satz 1] has shown that, for the localization $Z_{p}$ of $Z$ at $p$, the map $a_{Z_{p}}(G, \mathfrak{U}) \rightarrow a_{Z_{p}}^{*}(G, \mathfrak{U})$ induced by the completion functor is monic. Hence our proof transfers to $a_{Z_{p}}(G, \mathfrak{U})$. (Indeed, most of the results of [4] can now be taken with coefficients from $Z_{p}$.) Dress has further indicated to me in a private communication that he has a method of descent from $Z_{p}$ to $Z$. This method will be discussed in his forthcoming lecture notes from Universität Bielefeld.

\section{REFERENCES}

1. C. W. Curtis and I. Reiner, Representation theory of finite groups and associative algebras, 2nd ed., Interscience, New York, 1966.

2. A. Dress, On integral and modular relative Grothendieck rings, Multicopied Notes of the Summer Open House for Algebraists, Aarhus University, 1970, pp. 85-108.

3. —, Relative Grothendieckringe über semilokalen Dedekindringen, Surjektivität des Reduktionshomomorphismus und ein Theorem von Suan (to appear).

4. W. Gustafson, Integral relative Grothendieck rings, J. Algebra (to appear).

Department of Mathematics, Indiana University, Bloomington, Indiana 47401

Current address: Department of Mathematics, Brandeis University, Waltham, Massachusetts 02154 